\title{
Hydrogen Production via a Commercially Ready Inorganic Membrane Reactor
}

Semi-Annual Technical Progress Report

Reporting Period: October 1, 2006 to March 31, 2007

Paul K. T. Liu

Project Director

June 20, 2007

PREPARED FOR THE UNITED STATES

DEPARTMENT OF ENERGY

Under Cooperative Agreement

No. DE-FC26-03NT41852

By

MEDIA AND PROCESS TECHNOLOGY, INC.

1155 William Pitt Way

Pittsburgh, PA 15238 


\section{Disclaimer}

This report was prepared as an account of work sponsored by an agency of the United States Government. Neither the United States Government nor any agency thereof, nor any of their employees, makes any warranty, express or implied, or assumes any legal liability or responsibility for the accuracy, completeness, or usefulness of any information, apparatus, product, or process disclosed, or represents that its use would not infringe privately owned rights. Reference herein to any specific commercial product, process, or service by trade name, trademark, manufacturer, or otherwise does not necessarily constitute or imply its endorsement, recommendation, or favoring by the United States Government or any agency thereof. The views and opinions of authors expressed herein do not necessarily state or reflect those of the United States Government or any agency thereof. 


\section{$\underline{\text { Abstract }}$}

The commercial stainless steel (SS) porous substrate (i.e., $\mathrm{ZrO}_{2} / \mathrm{SS}$ from Pall Corp.) was evaluated comprehensively as substrate for the deposition of the CMS membrane for hydrogen separation. The CMS membrane synthesis protocol we developed originally for the ceramic substrate was adapted here for the stainless steel substrate. Unfortunately no successful hydrogen selective membranes had been prepared during Yr I of this project. The characterization results indicated two major sources of defect present in the stainless steel substrate, which may contribute to the poor CMS membrane quality. They include (i) leaking from the crimp boundary of the stainless steel substrate, and (ii) the delamination of the $\mathrm{ZrO}_{2}$ layer deposited on the stainless steel substrate during CMS membrane preparation. Recently a new batch of the stainless steel substrate (as the $2^{\text {nd }}$ generation product) was received from the supplier. Our characterization results confirm that leaking of the crimp boundary no longer exists. The thermal stability of the $\mathrm{ZrO}_{2} /$ stainless steel substrate under the CMS membrane preparation condition will be evaluated during the remaining period of the project. Our goal here is to determine the suitability of the $2^{\text {nd }}$ generation $\mathrm{ZrO}_{2} / \mathrm{SS}$ as substrate for the preparation of the CMS membrane for hydrogen separation by the end of this project period. 


\section{TABLE OF CONTENTS}

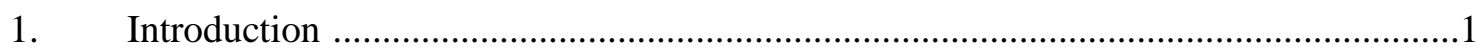

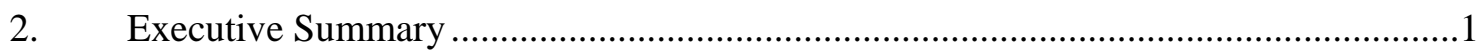

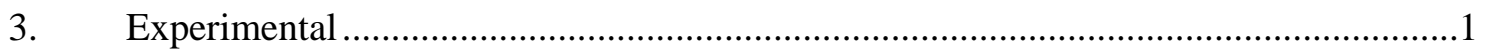

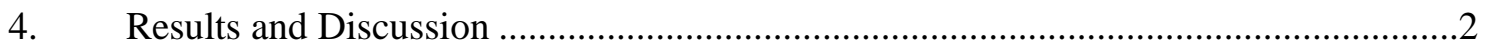

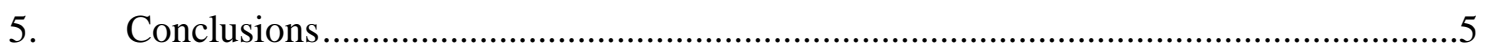

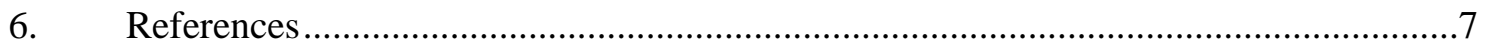

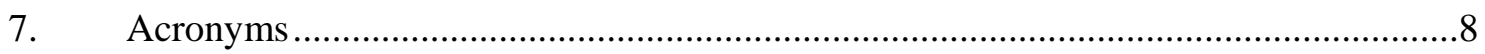




\section{List of Graphical Materials}

Figures

Figure 1 Illustration of the "crimped endseal” region where defect was identified with bubble test.

Figure 2 SEM Photomicrographs of the cross section of inside tube surface of $\mathrm{ZrO}_{2} / \mathrm{SS}$ substrate including $\mathrm{ZrO}_{2}$ layer and $\mathrm{M} \& \mathrm{P} \mathrm{Al}_{2} \mathrm{O}_{3}$ layer......................................... 4

Figure 3 SEM photomicrograph of the cross section of the $\mathrm{ZrO}_{2} /$ stainless steel substrate provided by the supplier as the $2^{\text {nd }}$ generation product..........................................5

\section{Tables}

Table 1 Comparison of gas permeation for CMS membranes deposited on stainless steel vs ceramic substrate. ....................................................................................... 2

Table 2 Qualitative description of defects observed on CMS membranes deposited on stainless steel substrate 


\section{Introduction}

During this 6 month extension period, we have focused on the two areas below:

i. Characterization of Pall's stainless steel substrates and the preparation of these substrates for the deposition of CMS membranes.

ii. $\quad$ Preparation of the final project report.

During the Year I of this project, part of our project was focused on the deposition of our CMS membrane on the commercial stainless steel (SS) substrate. CMS membranes prepared on this SS substrate were not satisfactory due to defects present in the substrate. During the last month of this extension period, the supplier provided the improved stainless steel substrate $\left(2^{\text {nd }}\right.$ generation). This report summarizes the very preliminary results obtained in this less than one month period. In addition, the final report has been under preparation, which is not included in this semi-annual report.

\section{Executive Summary}

The commercial stainless steel (SS) substrate (i.e., $\mathrm{ZrO}_{2} / \mathrm{SS}$ from Pall Corp.) was evaluated comprehensively as substrate for the deposition of the CMS membrane for hydrogen separation. The CMS membrane synthesis protocol we developed originally for the ceramic substrate was adapted to this SS substrate. Unfortunately no successful membranes had been prepared during Yr I of this project. Our characterization results indicated two major sources of defect present in the SS substrate, which may contribute to the poor quality of the CMS membrane. They include (i) leaking from the crimp boundary of the SS substrate, and (ii) the delamination of the $\mathrm{ZrO}_{2}$ layer deposited on the SS substrate during the preparation of the CMS membranes. Recently a new batch of the SS substrate as the $2^{\text {nd }}$ generation product was received from the supplier. Our characterization results confirm the defect of the crimp boundary no longer exists. The thermal stability of the $\mathrm{ZrO}_{2}$ /stainless steel substrate under the CMS membrane preparation condition will be evaluated during the remaining period of the project. Our goal here is to determine the suitability of the $\mathrm{ZrO}_{2} / \mathrm{SS}$ as substrate for the preparation of the CMS membrane for hydrogen separation.

\section{Experimental}

\subsection{Morphological Characterization of Stainless Steel Substrate}

SEM was performed on the cross section of the stainless steel substrate deposited with $\mathrm{ZrO}_{2}$ provided by Pall Corp.

\section{2. $\quad$ Characterization on Degree of Defect}

Bubble point has been performed for the substrates received recently. 


\subsection{Deposition of CMS Thin Film}

The $\mathrm{ZrO}_{2} / \mathrm{SS}$ substrate was deposited with the CMS membrane following the same protocol we have used throughout this project. This $\mathrm{CMS} / \mathrm{ZrO}_{2} / \mathrm{SS}$ membrane was then characterized with the SEM, the bubble point, and gas permeation as described above.

\section{Results and Discussion}

\section{1. $\quad$ Preparation of CMS/SS Membrane in Yr I}

During Yr I of this project, we received several samples of the first generation stainless steel substrate provided by our supplier, Pall Corp. The stainless steel substrate was deposited with $\mathrm{ZrO}_{2}$ to reduce its pore size to 0.1 micron range by the supplier. For us to deposit the CMS membrane, microporous $\mathrm{Al}_{2} \mathrm{O}_{3}$ thin film was deposited on top of this substrate to further reduce its pore size to the range comparable to the ceramic substrate we have used throughout our study.

This substrate with $\mathrm{ZrO}_{2}$ and $\mathrm{Al}_{2} \mathrm{O}_{3}$ deposition was then used for the deposition of the CMS membrane. It was found that the CMS membranes thus produced show much lower selectivity of H2 over other gases as shown in Table 1. It should be noted that the CMS/SS membrane quality became unacceptable when calcined at the intermediate and higher temperatures although these are the temperature we prefer to produce a CMS membrane with a high selectivity. For instance, $\mathrm{H}_{2} / \mathrm{N}_{2}$ of 39 and 98 at $220^{\circ} \mathrm{C}$ were obtained for the CMS/Ceramic membranes calcined at the intermediate and high temperatures. The selectivity for $\mathrm{H}_{2} / \mathrm{CH}_{4}$ is much higher. However, the best selectivity we have obtained for the CMS/SS membrane is $\sim 17$ for $\mathrm{H}_{2} / \mathrm{N}_{2}$ and $\mathrm{H}_{2} / \mathrm{CH}_{4}$ at $220^{\circ} \mathrm{C}$. Evidently the lower selectivity of CMS/SS is partially attributed to the lack of the thermal stability of the membrane when calcined at the intermediate and the higher temperature.

Table 1 Comparison of gas permeation for CMS membranes deposited on stainless steel vs ceramic substrate.

\begin{tabular}{|l|l|l|r|r|r|r|}
\hline $\begin{array}{c}\text { Part } \\
\text { ID }\end{array}$ & Substrate & \multicolumn{1}{c|}{$\begin{array}{c}\text { Firing } \\
\text { Temp. }\end{array}$} & $\begin{array}{c}\mathbf{H}_{\mathbf{2}} \text { Permeance } \\
{\left[\mathbf{m}^{\mathbf{3}} / \mathbf{m}^{\mathbf{2}} / \mathbf{h r} / \mathbf{b a r}\right]}\end{array}$ & $\begin{array}{c}\mathbf{H}_{\mathbf{2}} / \mathbf{N}_{\mathbf{2}} \\
\mathbf{2 0 0}^{\circ} \mathbf{C}\end{array}$ & $\begin{array}{c}\mathbf{H}_{2} / \mathbf{C H}_{\mathbf{4}} \\
\mathbf{2 0 0}{ }^{\circ} \mathbf{C}\end{array}$ & $\begin{array}{c}\mathbf{H}_{2} / \mathbf{C}_{\mathbf{2}}{ }^{-} \\
\mathbf{2 0 0} \mathbf{C}^{\circ}\end{array}$ \\
\hline DZp-18 & Pall S.S. & Low & 1.8 & 17 & 16.8 & 45 \\
\hline NN-02 & M\&P Ceramic & Intermediate & 1.6 & 39 & 155 & $>500$ \\
\hline DZ-216 & M\&P Ceramic & High & 1.0 & 98 & 166 & $>500$ \\
\hline
\end{tabular}


Table 2 Qualitative description of defects observed on CMS membranes deposited on stainless steel substrate

\begin{tabular}{|l|c|c|c|c|}
\hline Firing Temp & \# of Parts & \%Successful & \# of Crimp Leak & \# of Center/Delam. \\
\hline Low & 8 & 50 & 2 & 4 \\
\hline Intermediate1 & 2 & 0 & 2 & 1 \\
\hline Intermediate2 & 4 & 0 & 4 & 3 \\
\hline
\end{tabular}

\subsection{Morphological Characterization of CMS/SS Membranes}

The CMS/SS memrbranes prepared above were characterized morphologically with the bubble point method for the prupsoe of identifying defects of the membranes. Although this method does not provide the quantitative information on the number of defects, it does provide an overview on the quality of the membrane layer as summarized in Table 2. Out of the 14 parts we have prepared, we found that the CMS/SS membranes calcined at the low temperature deliver about 50\% successful rate, i.e., no obvious bubbles were identified. No successful tubes were identified for those calcined at the intermediate temperature. Further, most of the defects were found from the crimp end where the porous stainless steel was welded to the non-pervious stainless steel as end seals. Nearly all tubes calcined at the intermediate temperature show this type of defects. The other type of defects is the delamination of the CMS layer at the center of the membrane section. The defects are further elucidated in the next section.

\subsection{Characterization of Defects of CMS/SS Membranes}

Two types of defects were found as indicated in the above section. The first type defect is located at the crimp boundary between the solid stainless steel section and the porous stainless steel section as described in the schematic in Figure 1. The second type defect is resulted from the intermediate layer delamincation as presented in the SEM pictures in Figure 2. Firing temperatures required to produce highly $\mathrm{H}_{2}$ selective membranes result in delamiantion of the intermediate $\mathrm{ZrO}_{2}$ layer. Based upon the diagonosis above, we believe that the end seal defects are most likely present in the stainless steel substrate, while the delamincation is resulted from the lack of the thermal stability of the stainless steel/ $\mathrm{ZrO}_{2}$ substrate under the temperature/atmosphere required for the preparation of the CMS substrate. 


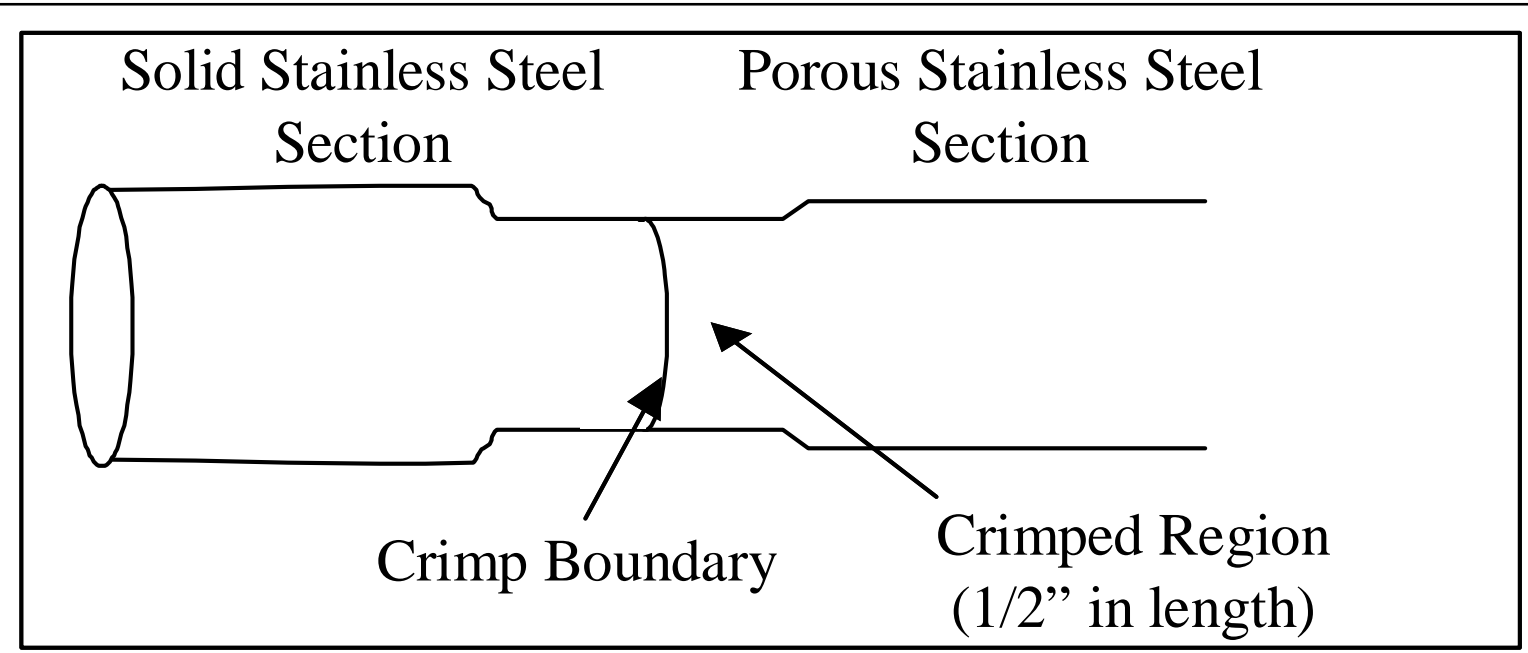

Figure 1 Illustration of the "crimped endseal” region where defect was identified with bubble test.

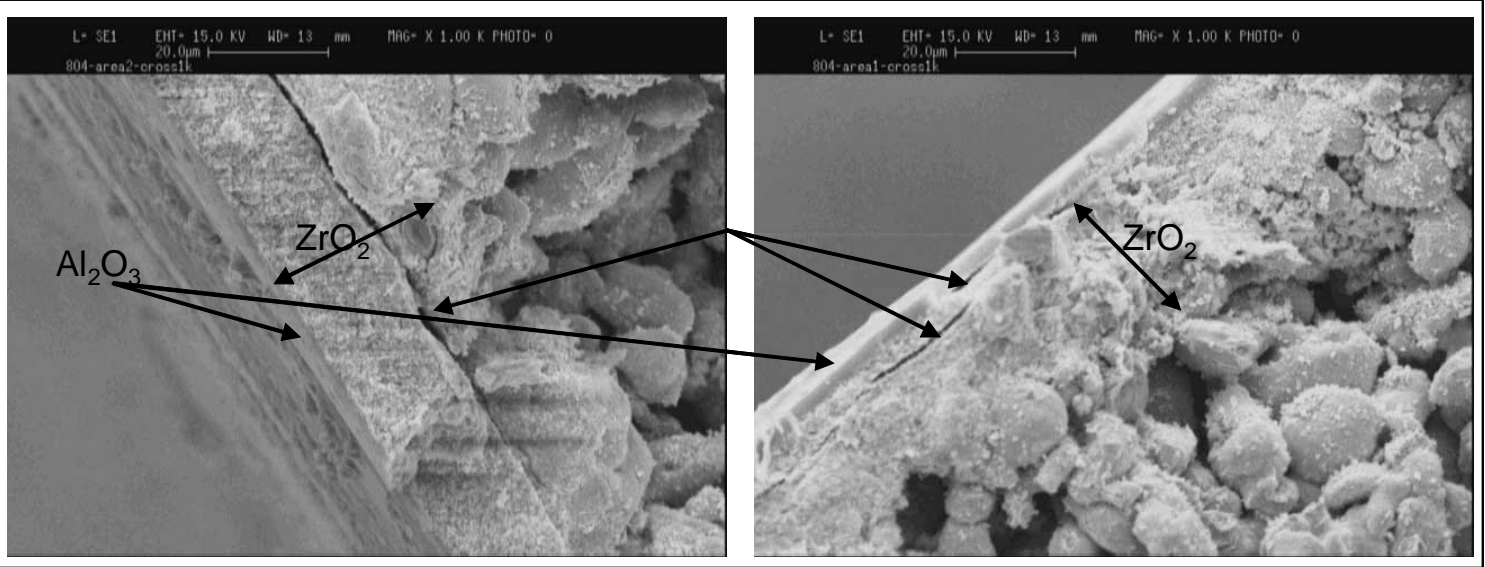

Figure 2

SEM Photomicrographs of the cross section of inside tube surface of $\mathrm{ZrO}_{2}$ /stainless steel substrate including $\mathrm{ZrO}_{2}$ layer and $\mathrm{M} \& \mathrm{P} \mathrm{Al}_{2} \mathrm{O}_{3}$ layer.

\section{4. $\quad$ Characterization of Stainless Steel Substrate received Recently}

Recently we have received the $2^{\text {nd }}$ generation of the $\mathrm{ZrO}_{2} / \mathrm{SS}$ substrate provided by Pall Corp with the improved end seals and other features. Thus, our CMS on SS substrate activity was resumed. Thus far, we have performed the SEM morphological characterization of the cross section of the membrane and the leak check of the end seals. Figure 3 presents the cross section 
of the $2^{\text {nd }}$ generation of $\mathrm{ZrO}_{2} / \mathrm{SS}$ substrate. It appears that the $\mathrm{ZrO}_{2}$ layer with 25 micron thickness was deposited on the stainless steel substrate. Moreover, the top surface of the $\mathrm{ZrO}_{2}$ appears very smooth. Bubble point was performed on one of the samples, Pall Id-"MP\&T-

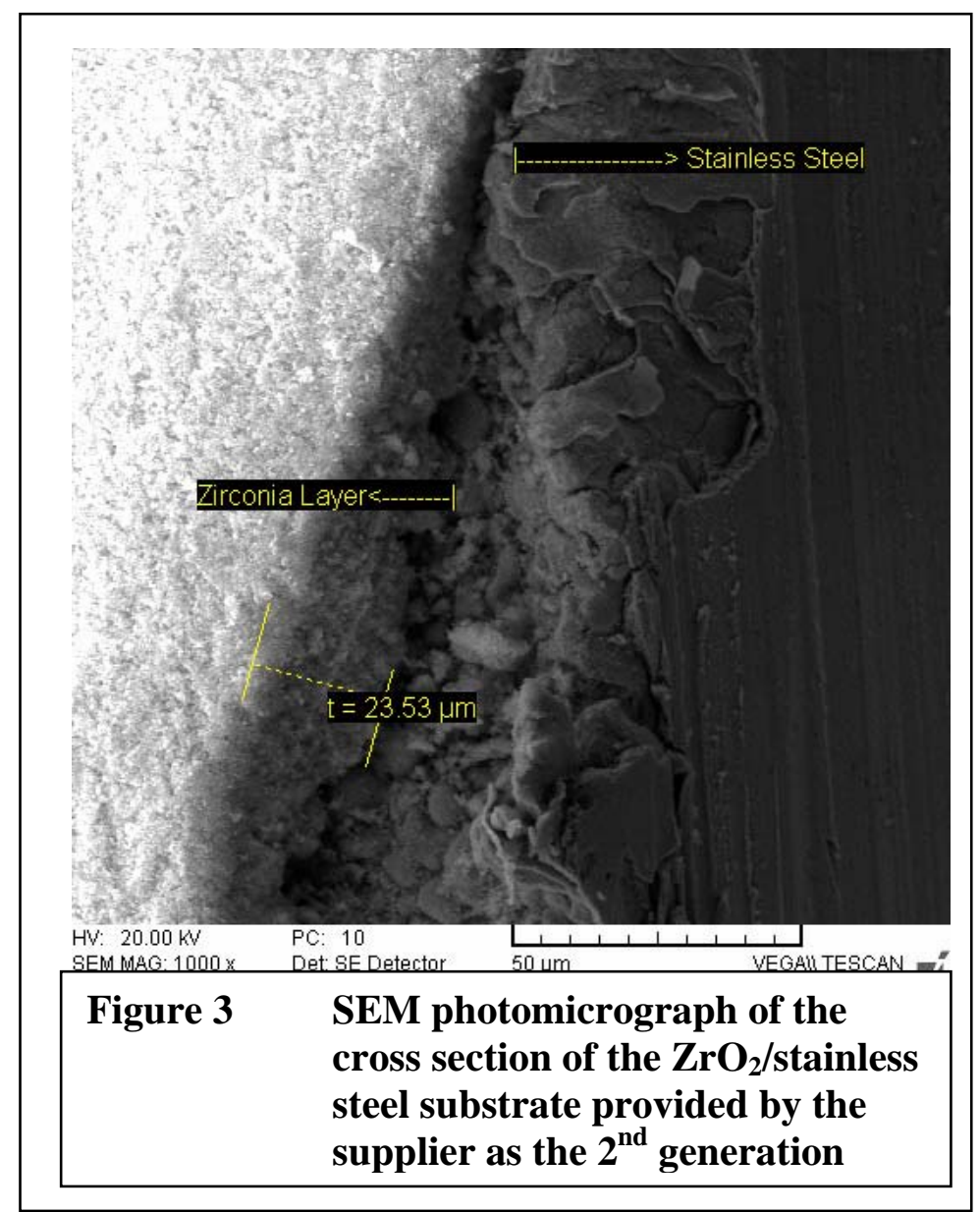

032707-4". No foaming/breakthrough was observed at 24 psi of IPA. Obviously, the welds/endseals looked good for this sample. The permeances at room temperature are 143 and $288 \mathrm{~m} 3 / \mathrm{m} 2 / \mathrm{hr} / \mathrm{bar}$ for He and $\mathrm{N}_{2}$ respectively. In summary, based upon our limited characterization result, it appears that defect of the end seals has been corrected for the $2^{\text {nd }}$ generation $\mathrm{ZrO}_{2} / \mathrm{SS}$ substrate. More samples will be characterized to obtain the statistically significant result. In addition, the thermal stability of these substrates will be tested during the remaining period of the project. Our goal here is to collect enough characterization result to confirm the suitability of the $\mathrm{ZrO}_{2} / \mathrm{SS}$ substrate for the deposition of the CMS membranes. No CMS membrane deposition activity will be attempted due to the limited time and budget remain for this project.

\section{Conclusions}

Our preliminary study on the deposition of the CMS membrane on $\mathrm{ZrO}_{2} / \mathrm{SS}$ substrate concludes: 
- The CMS membrane synthesis protocol we developed originally for the ceramic substrate was adapted to this SS substrate. Unfortunately no successful membranes had been prepared during Yr I. Our characterization results indicated two major sources of defect present in the stainless steel substrate, which contribute most likely the poor quality of the CMS membranes. They include (i) leaking from the crimp boundary of the SS substrate, and (ii) the delamination of the $\mathrm{ZrO}_{2}$ layer deposited on the stainless steel substrate during the CMS membrane preparation.

- $\quad$ Recently a new batch of the stainless steel substrate as the $2^{\text {nd }}$ generation product was received from the supplier. Our characterization results confirm the defect of the crimp boundary no longer exists.

The thermal stability of the $\mathrm{ZrO}_{2}$ /SS substrate under the CMS membrane preparation condition will be evaluated during the remaining period of the project. Our goal for the remaining project period is to determine the suitability of the $\mathrm{ZrO}_{2} / \mathrm{SS}$ membrane as substrate for the preparation of the CMS membrane for hydrogen separation. 
References:

None 


\section{List of Acronyms:}

SS: $\quad$ stainless steel

CMS: carbon molecular sieve 